\title{
Neural Integration of Risk and Effort Costs by the Frontal Pole: Only upon Request
}

\author{
Christopher J. Burke, ${ }^{1}$ Christian Brünger, ${ }^{2}$ Thorsten Kahnt, ${ }^{1}$ Soyoung Q. Park, ${ }^{1}$ and Philippe N. Tobler ${ }^{1}$ \\ ${ }^{1}$ Laboratory for Social and Neural Systems Research, Department of Economics, University of Zurich, 8006 Zürich, Switzerland, and ${ }^{2}$ Faculty of Psychology \\ and Neuroscience, Maastricht University, 6229 ER Maastricht, Netherlands
}

Rewards in real life are rarely received without incurring costs and successful reward harvesting often involves weighing and minimizing different types of costs. In the natural environment, such costs often include the physical effort required to obtain rewards and potential risks attached to them. Costs may also include potential risks. In this study, we applied fMRI to explore the neural coding of physical effort costs as opposed to costs associated with risky rewards. Using an incentive-compatible valuation mechanism, we separately measured the subjective costs associated with effortful and risky options. As expected, subjective costs of options increased with both increasing effort and increasing risk. Despite the similar nature of behavioral discounting of effort and risk, distinct regions of the brain coded these two cost types separately, with anterior insula primarily processing risk costs and midcingulate and supplementary motor area (SMA) processing effort costs. To investigate integration of the two cost types, we also presented participants with options that combined effortful and risky elements. We found that the frontal pole integrates effort and risk costs through functional coupling with the SMA and insula. The degree to which the latter two regions influenced frontal pole activity correlated with participant-specific behavioral sensitivity to effort and risk costs. These data support the notion that, although physical effort costs may appear to be behaviorally similar to other types of costs, such as risk, they are treated separately at the neural level and are integrated only if there is a need to do so.

\section{Introduction}

Adaptive decisions benefit from accurately assessing and computing any potential costs before pursuing a course of action. Two types of costs that may reduce future reward value are the physical effort required to obtain rewards, and the risk arising from variability in reward magnitudes. Although physical effort has been examined with respect to motivation and reward (Pessiglione et al., 2007; Schmidt et al., 2010; Cléry-Melin et al., 2011), few investigations have looked into the similarities of effort coding with other types of cost coding. Of these few, one is a study revealing differences in the processing of effort and delay costs when the two of them are presented separately (Prévost et al., 2010).

Previous studies show that animals are less likely to choose more effortful options when the size of the reward is fixed (Collier and Levitsky, 1968; Walton et al., 2009). Together with findings of risk and delay discounting (Richards et al., 1997; St Onge and Floresco, 2010), this suggests that effort impacts behavior similarly to other costs. Nevertheless, it remains an open question whether costs are coded in a common or distinct manner at the

Received July 31, 2012; revised 0ct. 20, 2012; accepted Nov. 25, 2012.

Author contributions: C.J.B. and P.N.T. designed research;C.J.B., C.B., T.K., S.Q.P., and P.N.T. performed research; C.J.B. and P.N.T. analyzed data; C.J.B., T.K., S.Q.P., and P.N.T. wrote the paper.

We thank Christoph Hollnagel and Adrian Etter for helpful discussions. This work was supported with funding from the Swiss National Science Foundation (PP00P1_128574) and the Swiss National Centre of Competence in Research in Affective Sciences.

Correspondence should be addressed to Christopher J. Burke, Laboratory for Social and Neural Systems Research, Department of Economics, University of Zurich, Blümlisalpstrasse 10, 8006 Zürich, Switzerland. E-mail: christopher.burke@econ.uzh.ch.

DOI:10.1523/JNEUROSCI.3662-12.2013

Copyright $\odot 2013$ the authors $\quad 0270-6474 / 13 / 331706-08 \$ 15.00 / 0$ neural level. Lesion studies suggest that distinct brain regions code effort and delay costs (Rudebeck et al., 2006; Walton et al., 2006; Floresco et al., 2008). However, neuroimaging studies in humans report conflicting findings of combined and distinct coding of effort with other economically relevant factors. Similar brain regions integrate effort with reward magnitude (Croxson et al., 2009) and probability (Treadway et al., 2012). In contrast, effort and delay costs are coded in anatomically separate areas (Prévost et al., 2010). One possible explanation for these conflicting findings could be that the different modalities of cost and reward are either presented in isolation on single trials (Prévost et al., 2010) or in a compound fashion within a single trial (Croxson et al., 2009; Treadway et al., 2012). Here, we investigate this possibility by using both isolated and compound trials. Reconciliation for the conflicting findings could be common coding in compound trials but separate coding in isolated trials.

We hypothesized that effort costs would be reflected in the sensorimotor integration areas of the cingulate cortex, supplementary motor area, and the striatum (Pessiglione et al., 2007; Croxson et al., 2009; Kurniawan et al., 2010; Prévost et al., 2010). In contrast, costs associated with risky options would be reflected in risk-responsive areas, such as the insula (Preuschoff et al., 2008; Mohr et al., 2010; Burke and Tobler, 2011). In comparing isolated and compound trials, we considered two possibilities. A common cost-coding region would respond to costs in both isolated and compound trials, regardless of cost modality. Alternatively, distinct regions may code their specific cost type during isolated trials and feed into some third region only during compound trials, when there is need to combine cost types. Regions that have been assigned such an integrative role include the an- 
terior cingulate (Croxson et al., 2009; Kennerley et al., 2009) and frontal pole (Prabhakaran et al., 2000; Bunge et al., 2009; Wunderlich et al., 2011).

\section{Materials and Methods}

Participants and prescanning procedures. Twenty-three participants (mean age, 23.5 years; SD, 4.7 years; 8 women; all right-handed) took part in the experiment. Of the 23 participants scanned, 19 entered further fMRI analysis. One participant was excluded due to normalization problems during preprocessing, one was excluded due to incidental findings, and two participants were excluded for excessive head movement during the task. All participants had normal or corrected-to-normal vision and were screened to exclude those with a previous history of neurological or psychiatric disease. All gave informed consent and the study was approved by the Research Ethics Committee of the Canton of Zurich.

An MR-compatible isometric dynamometer (Zühlke Engineering and Sensory-Motor Systems Laboratory) was used to assess participant handgrip force and allow participants to exert effort. First, participants were asked to grip the $600 \mathrm{~N}$ dynamometer with their left hand and perform five maximal-strength compressions. During this phase, the raw force readout from the dynamometer was displayed as a thermometer level on the computer screen and participants were instructed to try to fill the thermometer while the words "MAX GRIP" appeared on the screen for $2 \mathrm{~s}$ each time. The average of these five force readings was used to calibrate the display and set the $100 \%$ effort levels of every participant for the main task. The variance of the five maximal-force readings was used to calibrate thresholds around the effort levels presented later. These thresholds served to minimize the risk that a certain effort level may not be attained by the participant due to behavioral noise. After this calibration, participants were invited to read the task instructions.

Task instructions and participant payment. Participants were informed that they were taking part in an experiment to investigate how people value effortful and risky prospects. The instructions covered the meanings of the different stimulus types, the incentive-compatible BeckerDeGroot-Marschak (BDM)-like valuation stage and how payment was calculated. Understanding of the various stages of the task was verified during the instruction phase through a series of questions and training trials.

At the start of each trial, the participant was endowed with 1 Swiss franc, which he or she could spend on that trial. Participants would then be presented with an option that had an expected value of 50 cents (one hundredth of a franc is called 1 Rappen in German-speaking parts of Switzerland. For the reader's benefit, we refer to 1 Rappen as 1 cent). However the level of effort required to gain the 50 cents, or the level of risk associated with it, varied from trial to trial. During option presentation, the participants were instructed to think about how much the option is worth to them.

The participants could then choose the "pay-to-play" option by determining how much of their single trial's endowment to spend (in steps of 1 cent each) and entering this as their bid. The task computer would then randomly select a number between 0 and 100. If the participant's bid was higher than this random number, the participant would be able to play the option (i.e., exert the level of effort indicated by the option to gain 50 cents, or play the lottery with the indicated risk). Because the higher the participant bids, the more likely it is that they play the option, the optimal strategy in this auction is to bid the true subjective value of the option. Participants were instructed that in the event their bid was higher than the computer's, they would buy the option at the price of the computer's bid and gain the result of the option. Earnings were calculated for each session of the experiment. After they came out of the scanner, participants rolled a die to determine which session was finally paid out.

Stimuli and effort/risk levels. Three basic stimulus types were used in the experiment. These corresponded to isolated effortful, isolated risky, and compound options (Fig. 1B). Options were represented by blue rectangles. Effortful options were indicated by a green line across the rectangle, with the height of the green line corresponding to the required effort level $(0,25,50,75$, or $100 \%$ of the participant's maximal grip force). Risky options consisted of binary lotteries with equiprobable out- comes and were denoted by red lines across the rectangle, with the distance between the lines corresponding to the level of risk $(0,10,25,40$, or 50 cents SD around the expected value of 50 cents). Compound options had both green and red lines. Thirteen compound options were used (Table 1). Presentations of the three option types (isolated effort, isolated risk, and compound) were randomly intermixed during the experiment.

Task. Each trial started with a variable intertrial interval (ITI) with only the fixation cross visible in the center of the screen (Fig. $1 \mathrm{~A}$ ). ITI duration varied according to a truncated Poisson distribution with a range of 2-11 s. The ITI was followed by the presentation of an isolated or compound option in the center of the screen for $3 \mathrm{~s}$. The following screen consisted of the valuation stage $(2.5 \mathrm{~s})$, during which a horizontal scale ranging from 0 to 100 cents was presented below the fixation cross. Participants moved a red cursor along the length of this scale using a $100 \mathrm{~N}$ dynamometer in their right hand. Where the cursor was after $2.5 \mathrm{~s}$ was taken to be the subjective valuation of the preceding option. To remove the correlation between starting point and value, the cursor's starting point was randomly switched between 0 and 100 cents on the scale. The current value according to the position of the cursor on the scale was presented in real time in numbers between the scale and the fixation cross. During this time, the task computer selected a random integer between 0 and 100 .

Following the valuation phase, participants were presented with the fixation cross for a randomly jittered period of time (1-2s). The outcome phase followed, lasting 2 s. If the participant's bid was equal to or exceeded the randomly selected number, the option was realized (i.e., the option was resolved). In the case of isolated effortful options, the participant was required to reach the necessary grip force within $1 \mathrm{~s}$ and hold it for the remainder of the outcome phase. Failure to do so resulted in a red cross above the stimulus. Success was indicated by a green check mark above the stimulus, and participants received the 50 cent reward, minus the computer's randomly generated bid. In the case of a failure, participants did not win the 50 cents and lost their bid. In the case of isolated risky options, the stimulus was presented for $1 \mathrm{~s}$ and the randomly determined outcome was then enclosed in a white box for the remainder of the outcome phase. For compound options, the participant had to reach the required effort within $1 \mathrm{~s}$ and hold the level for the remainder, during which the randomly determined outcome of the risky outcome was enclosed in a white box.

In cases where the participant's bid did not equal or exceed the randomly drawn number, a blue rectangle with a red cross through it was displayed for $2 \mathrm{~s}$ (i.e., the option was unresolved). The participants kept their bid in this case. This was the same for all trial types (isolated effort, isolated risk, or compound). The outcome phase was then followed by the ITI. To familiarize themselves with the task and BDM procedure before scanning began, participants performed a short practice session consisting of one presentation of each trial type. Over the course of the experiment, each participant performed 60 isolated effort trials, 60 isolated risk trials, and 156 compound trials.

Data acquisition. Images were acquired using a Philips Achieva $3 \mathrm{~T}$ whole-body scanner with an eight-channel sensitivity-encoding head coil (Philips Medical Systems) at the Laboratory for Social and Neural Systems Research, University Hospital Zurich. The task was projected on a display, which participants viewed through a mirror fitted on top of the head coil. We acquired gradient echo $\mathrm{T} 2^{*}$-weighted echo-planar images (EPIs) with blood-oxygen-level-dependent (BOLD) contrast (slices/volume, 37; repetition time, 2 s). We collected 380-450 volumes in each session of the experiment, together with five "dummy" volumes at the start and end of each scanning session. Participants each completed four sessions of the experiment in the scanner, with short breaks between each session. Scan onset times varied randomly relative to stimulus onset times. A T1-weighted 3D turbo field echo structural image was also acquired for each participant. Volumes were acquired at a $+15^{\circ}$ tilt to the anterior commissure-posterior commissure line, rostral more than caudal. Imaging parameters were the following: echo time, $30 \mathrm{~ms}$; field of view, $220 \mathrm{~mm}$. The in-plane resolution was $2.75 \times 2.75 \mathrm{~mm}$, with a slice thickness of $3 \mathrm{~mm}$ and an interslice gap of $0.5 \mathrm{~mm}$. High-resolution T1-weighted structural scans were coregistered to their mean EPIs and averaged together to permit anatomical localization of the functional activations at the group level. 
A

\section{Effort Trials}
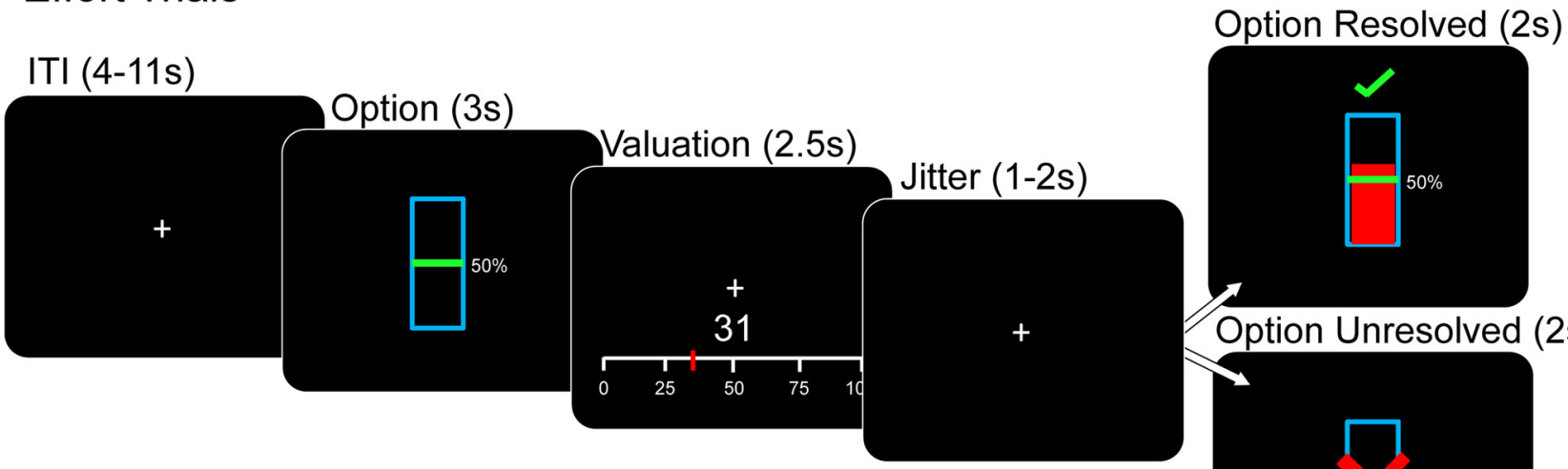

\section{Risk Trials}

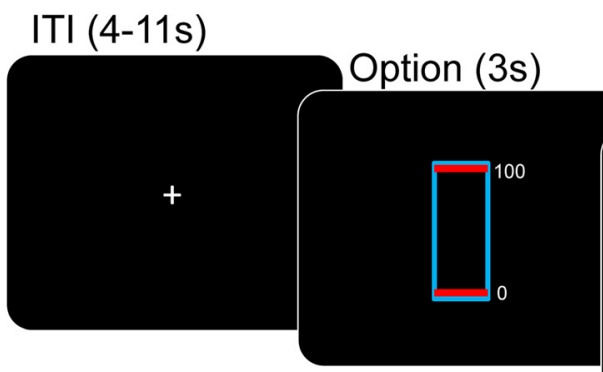

B
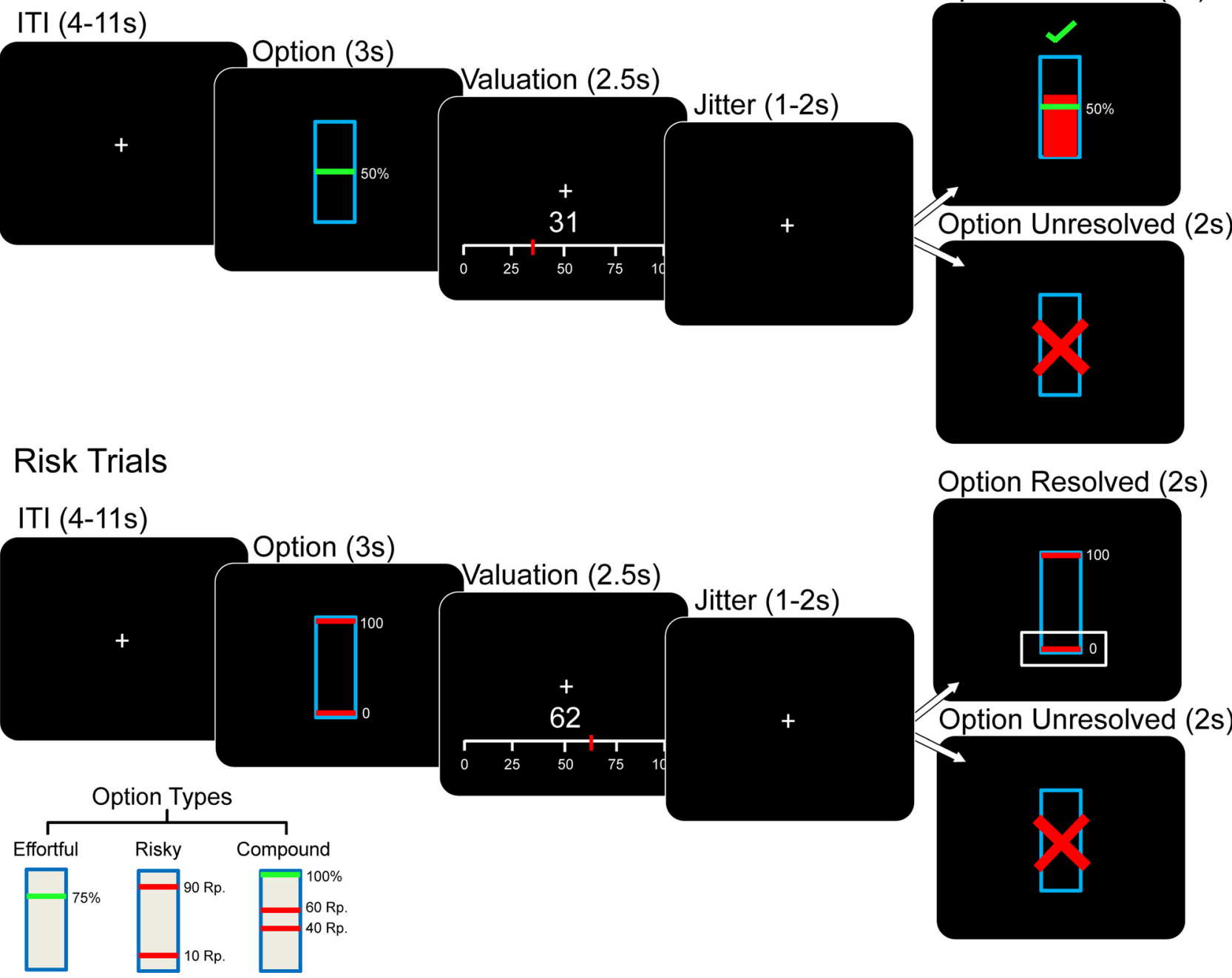

Figure 1. Experimental design. A, Task. Participants first viewed a fixation cross for a variable ITI of 4-11 s. At the end of the ITI, an option was presented for $3 \mathrm{~s}$. After option presentation, participants were required to value the option by moving a red cursor along a scale to enter a bid. The current bid (based on the position of the cursor) was presented in text above the scale. Participants held the cursor at their desired bid. This valuation phase lasted $2.5 \mathrm{~s}$ and measured willingness to pay for every option type. After a random jitter of $1-2 \mathrm{~s}$, the outcome phase was displayed for $2 \mathrm{~s}$. If the bid was above a randomly drawn number, the option was resolved and executed for the participant to gain monetary reward. $\boldsymbol{B}$, Examples of stimuli corresponding to different option types. Options were represented as a blue rectangle and could be divided into three basic subtypes. For effortful options, the required amount of physical effort was denoted by the height of a green bar ( 5 levels). Risky trial types were denoted by two red bars, the distance between which denoted the SD (i.e., risk) of the binary lottery ( 5 levels). Compound options contained risky (red bars) and effortful (green bars) elements, following the same convention as isolated effort and risk trials.

Table 1. Effort and risk levels used in compound options

\begin{tabular}{|c|c|c|c|c|c|c|c|c|c|c|c|c|c|}
\hline Effort level (percentage of maximum grip) & 0 & 0 & 25 & 20 & 25 & 50 & 50 & 50 & 75 & 80 & 75 & 100 & 100 \\
\hline Risk level (SD cents) & 0 & 50 & 10 & 25 & 40 & 10 & 25 & 40 & 10 & 25 & 40 & 0 & 50 \\
\hline Objective cost level (effort plus risk) & 0 & 4 & 2 & 3 & 4 & 3 & 4 & 5 & 4 & 5 & 6 & 4 & \\
\hline
\end{tabular}

Image analysis. We used a standard rapid-event-related fMRI approach in which evoked hemodynamic responses to each event type are estimated separately by convolving a canonical hemodynamic response function with the onsets for each event and regressing these against the measured fMRI signal. Statistical parametric mapping (SPM5; Functional Imaging Laboratory, University College London) served to spatially realign functional data, normalize them to a standard EPI template, and smooth them using an isometric Gaussian kernel with a full width at half maximum of $12 \mathrm{~mm}$.

Onsets of task events were modeled as separate $\delta$ functions and convolved with a canonical hemodynamic response function. Each trial type (i.e., each level of effort, risk, and combination of the two) was modeled with a separate regressor at the time of the presentation of that option. Separate regressors modeled the BDM valuation time, resolved isolated effortful options (i.e., effort exertion), unresolved isolated effortful options, resolved isolated risky options (i.e., experienced risk), unresolved isolated risky options, resolved compound options, and unresolved compound options. Trials where participants failed to reach the required effort level were not modeled separately, as these trials only differed in the option resolution task epoch, which was modeled across all trial types in the resolved effortful option and resolved compound option regressors. Across the whole experiment, subjects failed to reach the required effort level on $8.84 \%$ of effort and compound trials. In contrast, they always gave an adequate response in the valuation phase of the task, which was 
the main interest of the present study. Participant-specific movement parameters ( 3 regressors for rotations and 3 for translation) were also modeled as regressors of no interest.

To assess effort coding, we formed contrasts that used the individually determined subjective costs of each of the isolated five effort levels. For risk and compound trials we proceeded similarly, taking the subjective costs of each of the five risk levels and the subjective costs across the 13 compound option types as contrast weights on the single subject level. These contrasts were mean-corrected and captured the subjective sensitivity to changes in costs for effort, risk, and compound conditions on an individual basis. They were taken up to the second group level in simple $t$ tests to identify regions that code subjective sensitivity to changes in costs in the whole group of participants. Anatomically defined smallvolume correction was used to control for multiple comparisons in predefined regions of interest according to the boundaries defined in the Automatic Anatomical Labeling atlas. For presentation purposes, imaging results are shown at the threshold of $p<0.005$ uncorrected unless where specifically mentioned in the figure legends.

Time course extraction for psychophysiological interaction analysis. We extracted time courses across the whole experiment in the left and right insula (regions coding risk costs in isolated risky trials), in the ventral supplementary motor area (SMA) and posterior putamen (pPut) (regions coding effort costs in isolated effortful trials), and in the frontal pole (coding the value of compound options). To be sure the time courses did not contain spurious artifacts, we used the time course residuals after regressing out constant and linear terms, a whole-brain time course, and movement parameters.

\section{Results}

\section{Behavior}

To assess the subjective cost of effort and risk for each participant, we measured their willingness to pay for different levels of risk or effort, keeping the expected value constant. More specifically, in an incentive-compatible BDM-like auction mechanism, participants were required to bid for isolated effortful, isolated risky, and compound effortful and risky options that led to a monetary outcome. The BDM mechanism is designed such that the optimal strategy for subjects is to reveal their true willingness to pay (a proxy of subjective value) for options (Bohm et al., 1997). Crucially, all options, regardless of whether they entailed risk, effort, or both, had the same expected value of 50 cents. Effortful options consisted of having to squeeze with $0,25,50,75$, or $100 \%$ of maximal grip force to obtain 50 cents. Risky options consisted of the following lotteries: 50 cents (no risk); 60 or 40 cents; 75 or 25 cents; 90 or 10 cents; 100 or 0 cents (maximal risk). Compound options consisted of 13 orthogonal combinations of the two (Table 1). In the present study, effort-averse participants will always bid below the objective value of the option (50 cents) and decrease their bids as effort increases. The same would hold true with respect to risk for risk-averse participants. We calculated the subjective costs associated with each option by subtracting each participant's average willingness-to-pay for the option from its objective expected value.

\section{Isolated trials containing only effortful or risky options}

A one-way repeated-measures ANOVA was conducted to compare the effect of effort-cost level on willingness to pay. As expected, the proposed effort-cost level had a significant effect on willingness to pay $\left(F_{(4,72)}=6.52, p<0.001\right)$. There was a significant linear effect of effort cost on the subjective costs of the options $(\beta=1.79, t=3.44, p=0.002,14$ of 19 subjects showed increasing costs with increasing levels of effort) (Fig. $2 A$ ). There was also a significant effect of the level of effort on the postexperiment stimulus pleasantness ratings of effort cues and the level of effort required $\left(F_{(4,64)}=11.93, p<0.0001\right)($ Fig. $2 B)$ as well as a significant linear effect of the effort cost on pleasantness ratings $(\beta=-0.88, t=2.94, p=0.009)$. Thus, effort costs reduced both willingness to pay and pleasantness ratings. There was no significant shift in bidding behavior across time for effort trials ( $p=$ 0.87 , multiple regression with time and trial type as predictors). Required effort had no significant effect on error rates (failure to achieve the required effort), supporting the notion that there was no intrinsic risk during effort trials (repeated-measures ANOVA, $\left.F_{(3,54)}=1.02, p=0.39\right)$.

In a similar fashion, risk costs affected willingness to pay (repeated-measures ANOVA, $F_{(4,72)}=9.50, p<0.0001$ ) (Fig. $2 C)$, indicated by a significant linear effect of risk on the subjective costs of the options ( $\beta=3.06, t=3.06, p=0.007,15$ of 19 subjects showed increasing costs with increasing levels of risk). Also, the postexperiment stimulus pleasantness ratings of cues were affected by risk costs $\left(F_{(4,64)}=9.33, p<0.0001\right)$ (Fig. $2 D$ ) showing a significant linear effect of risk on pleasantness ratings $(\beta=-0.60, t=2.51, p=0.02)$. There was no significant shift in bidding behavior across time for risk trials $(p=0.58)$. Together, both risk and effort costs decreased the value of options as measured by willingness to pay or pleasantness rating.

\section{Compound trials containing both effortful and risky options}

Compound options contained both effortful and risky elements and evenly covered the effort-risk space of isolated options (Table 1). The example illustrated in Figure $1 B$ would indicate to the participant that $100 \%$ effort is required to see the outcome of the binary lottery (either 60 or 40 cents). There was a significant effect of objective cost (i.e., proposed level of effort plus proposed level of risk) on participants' willingness to pay for options (1-way repeated-measures ANOVA, $\left.F_{(6,108)}=19.49, p<0.0001\right)$, but no effect of time $(p=0.67)$. As expected, subjective costs increased as the objective compound costs increased (linear regression, $R^{2}$ $=0.54, p<0.01)$. The surface plot in Figure $2 E$ illustrates that costs increased as a function of both risk and effort in compound trials.

To further investigate how participants arrived at valuations during compound option presentation, we also tested whether the willingness to pay for isolated effortful and isolated risky trials could be used to predict the willingness to pay for compound options. We hypothesized that the subjective costs that each participant attached to the levels of effort and risk in isolated effortful and risky trials could be added in a linear fashion to arrive at the subjective cost for the various levels of compound options. To test this hypothesis, we performed a multiple linear regression that included individual effort costs and individual risk costs. Both effort $(\beta=0.48, t=3.91, p=0.001)$ and risk $\operatorname{cost}(\beta=0.75$, $t=9.75, p<0.001)$ regressors were significant. Across subjects, there was no significant difference between the relative contributions of effort and risk costs to compound option costs ( $p=$ 0.07). Indeed, participant's willingness to pay in isolated effortful and risky trials significantly predicted their willingness to pay in compound trials $(p<0.001$, Fig. $2 F)$. Thus, participants appeared to process and integrate both types of costs in compound trials in an additive fashion.

\section{Neuroimaging results \\ Areas sensitive to changes in the subjective costs of isolated effortful options}

To assess brain regions sensitive to changes in the costs of isolated effortful options, we looked for activity correlating with the mean-corrected participant-specific behavioral costs associated 
with the five levels of effort at the time of the option presentation in our a priori regions of interest. In agreement with our original hypothesis, activity in the ventral supplementary motor area (vSMA) reflected the changes in the subjective costs of effortful options (Fig. $3 A$; peak at $-9,8$, $52 ; Z=3.42 ; p<0.05$ corrected for SMA). Time course analysis revealed that the average BOLD response in the supplementary motor area region of interest differentiated between high-effort and low-effort options, with the maximal response occurring $\sim 8-10 \mathrm{~s}$ after the stimulus onset (Fig. 3B).

In addition to the vSMA, increasing cost of effort was also reflected in increased activity in motor-related regions of the striatum, such as the pPut (Fig. $3 A$; peak at $33,-10,-2 ; Z=3.49 ; p<0.05$ corrected for bilateral putamen). Time course analysis revealed that the average BOLD response in the right putamen differentiated between objectively higheffort and low-effort options in a similar manner to the vSMA (Fig. 3C). Thus, activity in both the vSMA and the pPut reflected the behavioral finding of higher subjective costs attached to options requiring increasing levels of effort. Comparisons between the subjective effort costs from the BDM auction and objective linear contrasts for effort showed no significant differences in fit for both vSMA $(t=0.70, p=0.49)$ and pPut $(t=1.33$, $p=0.19)$ (anatomical ROIs). One potential explanation for this similarity is that effort levels were determined subjectively during the calibration phase to adjust for across-participant differences in maximum strength.

In our last region of interest, the cingulate cortex, activity during option presentation correlated with the subjective cost of effortful options in two distinct clusters (anterior cingulate cortex peak at $-6,14$, 28; $Z=3.02 ; p<0.001$ uncorrected; midcingulate cortex peak at $9,-28,46$; $Z=3.23, p<0.001$ uncorrected). However, neither of these clusters survived small-volume correction for the cingulate cortex.
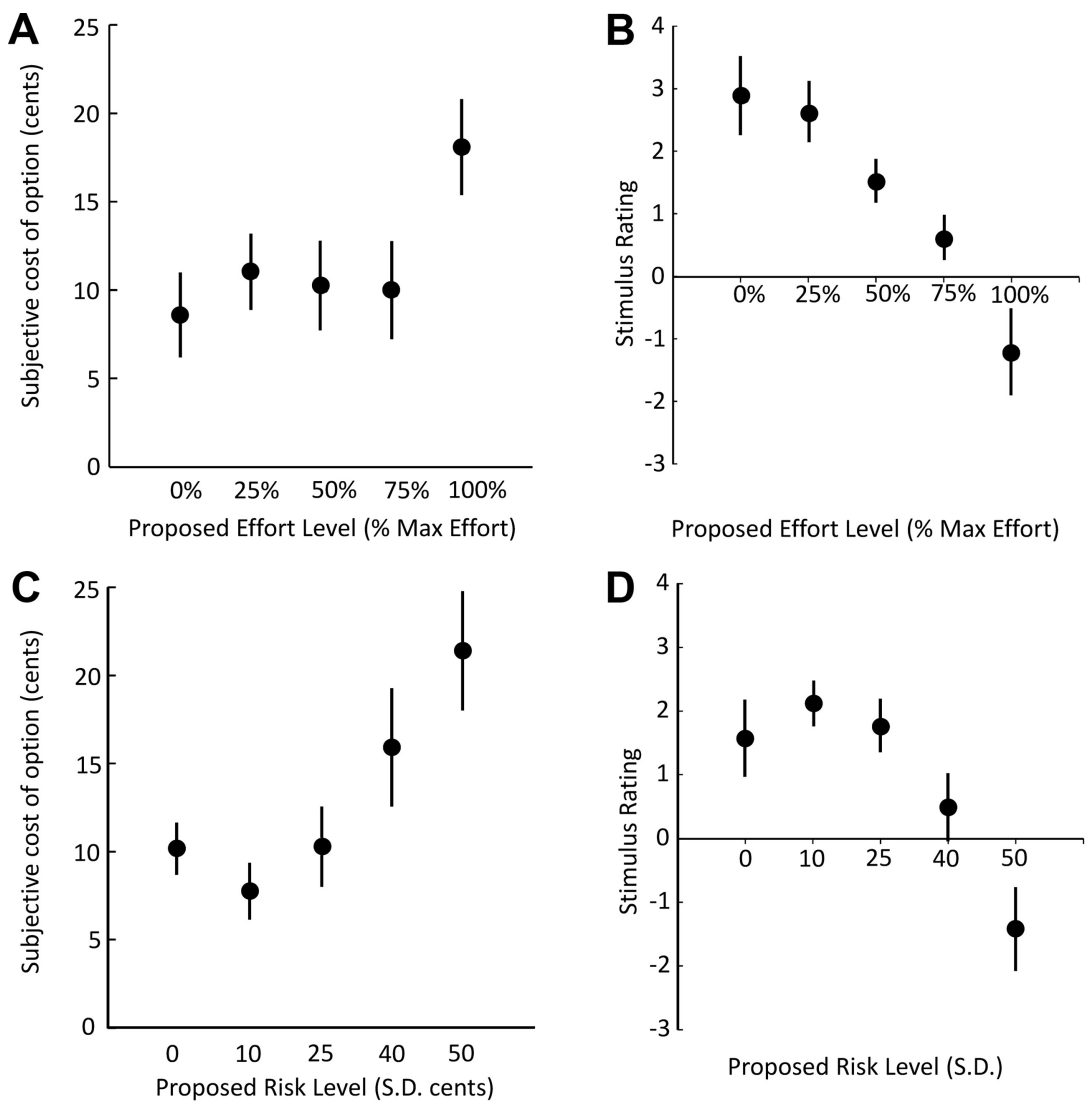

Proposed Effort Level (\% Max Effort)

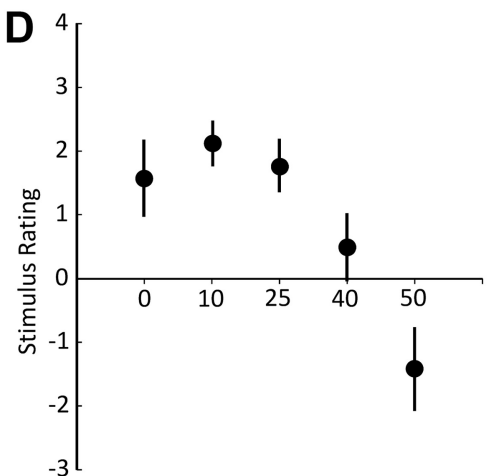

Proposed Risk Level (S.D.)
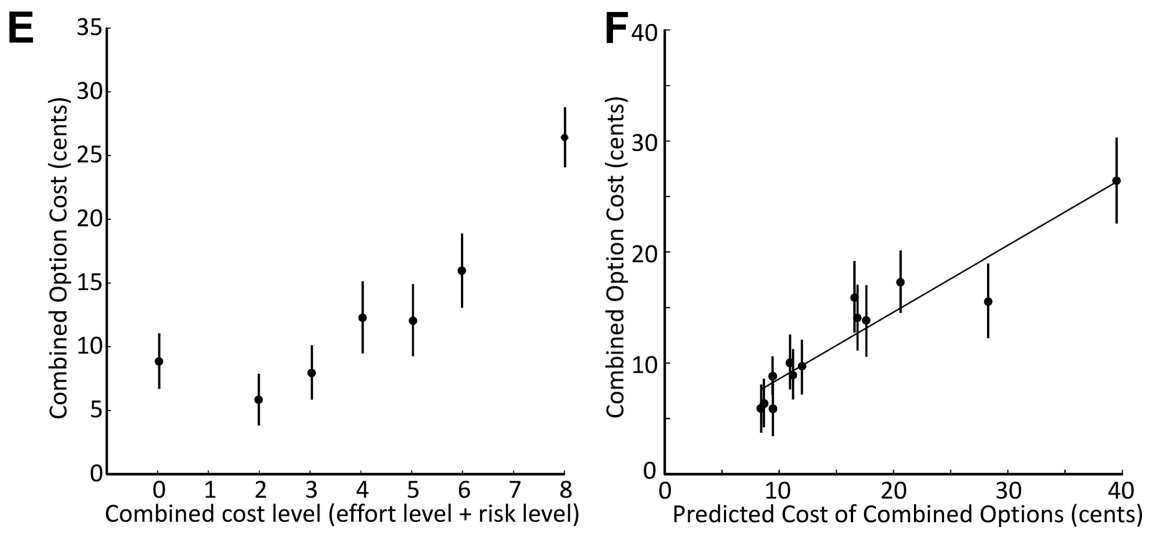

Figure 2. Behavioral results. $\boldsymbol{A}$, The subjective costs of options presented on isolated effort trials increased as a function of effort $(p<0.001)$, with participants judging the $100 \%$ effort option to be significantly more costly than all others $(p<0.01) . \boldsymbol{B}, A$ similar result was found for postexperiment pleasantness ratings of the effortful stimuli. $\boldsymbol{C}, 0$ n average, participants were risk averse, with the subjective cost of the risky options increasing as a function of the variance of the proposed lottery $(p<0.0001)$. $\boldsymbol{D}$, This result was also reflected in decreasing postexperiment pleasantness ratings of the risky stimuli as a function of risk. $\boldsymbol{E}$, Surface plot of the subjective costs of compound options. Compound options contained both effortful and risky elements, and the subjective costs associated with each option increased as a function of the additive objective levels of risk and effort $(p<0.01)$. $\boldsymbol{F}$, Using participants' subjective costs on isolated effort and isolated risk trials, we could significantly predict the subjective costs on compound trials $(p<0.001)$. All error bars represent the standard error of the mean.

\section{Areas sensitive to changes in the} subjective costs of isolated risky options

Next, we used the mean-corrected participant-specific behavioral costs to assess risk-related brain activation during risky option presentation. As hypothesized, activity in both right and left insula reflected the sensitivity to increasing costs of risk in a participant-specific manner (Fig. $4 A$; left insula peak at $-30,17$, $-14 ; Z=3.48 ; p<0.05$; right insula peak at $42,20,1 ; Z=3.35$; $p<0.05$; both left and right clusters small-volume corrected for insula). To further illustrate the effects, we plotted each partici- pant's $\beta$ estimate for an objective high-risk greater than low-risk contrast against the differences in subjective cost across these two options. As expected, this resulted in correlations for both right and left insula (Fig. 4B). Thus, insula activity scales with the subjective costs of risk as implemented in isolated options. Comparisons between the subjective risk costs from the BDM auction and objective linear contrasts for risk showed that insula activity (anatomical ROI) was significantly better explained by subjective risk costs $(t=2.57, p=0.01)$. 
A

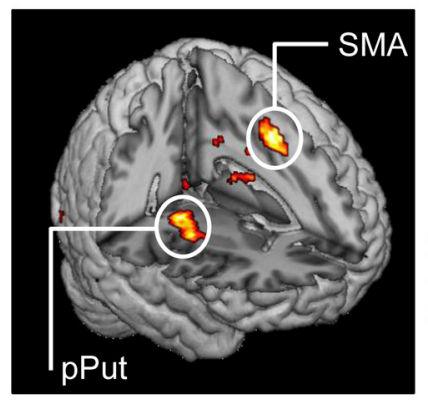

B

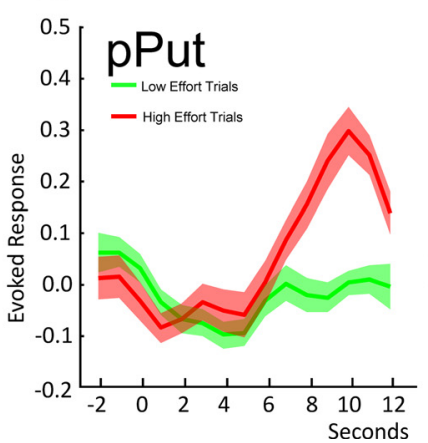

C

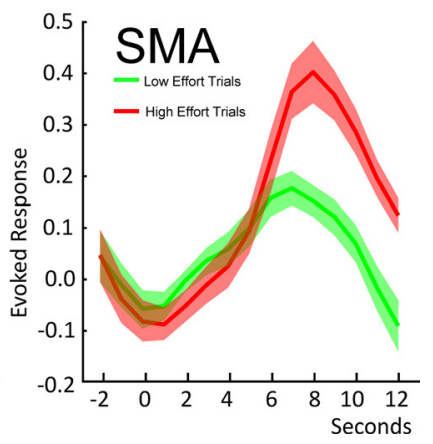

Figure 3. BOLD responses on isolated effort trials during option presentation. $A$, Neural activity in the pPut (peak at $33,-10,-2 ; Z=3.49 ; p<0.05$ corrected for bilateral putamen) and vSMA (peak at $-9,8,52 ; Z=3.42 ; p<0.05$ corrected for SMA) reflected the effort costs on isolated effort trials. $B$, Time course of activity in the vSMA (time locked to the presentation of options) in response to 100 and $75 \%$ effort trials (red line) and 0 and $25 \%$ effort trials (green line). C, A similar time course of the BOLD response to high-effort and low-effort trials (time-locked to the presentation of options) was also present in the pPut.

A

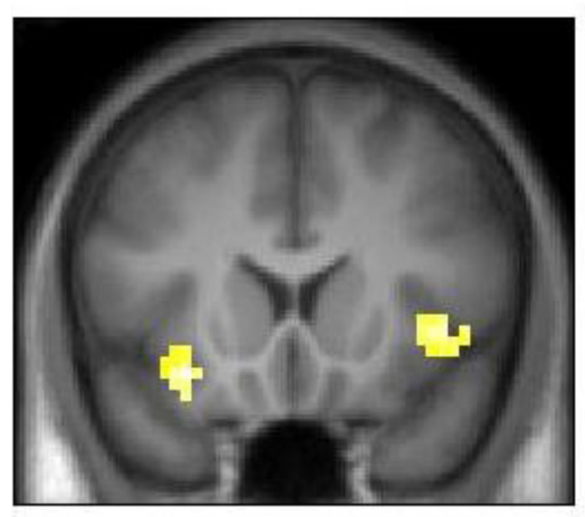

B

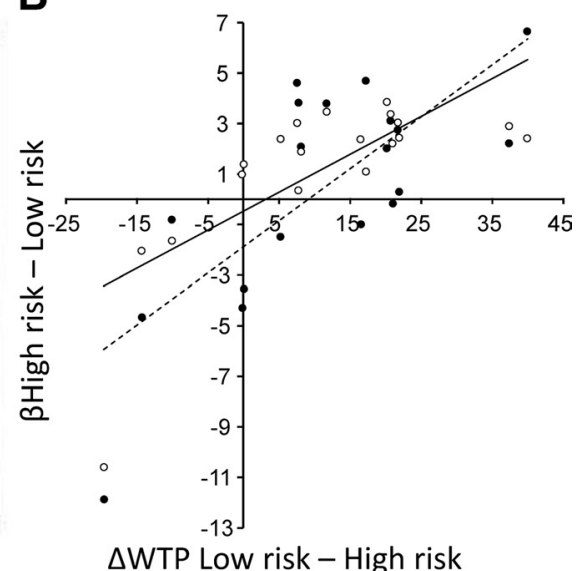

Figure 4. BOLD responses on isolated risk trials during option presentation. $A$, Activity in the left insula (peak at $-30,17,-14$; $Z=3.48 ; p<0.05$ ) and right insula (peak at $42,20,1 ; Z=3.35 ; p<0.05$; both clusters small-volume corrected for insula) reflected the subjective costs of risky options at the time of option presentation. $\boldsymbol{B}$, The degree of activation to the objective high-variance greater than low-variance lottery during option presentation increased as a function of individual risk aversion in both left insula (empty markers, solid line) and right insula (black markers, dashed line).

\section{Areas correlating with the subjective costs of isolated effortful and risky options}

To test whether there is a common brain region that codes sensitivity to changes in both effort costs and risk costs, we performed a conjunction analysis using the subjective cost contrast images from effort trials and risk trials. In our a priori regions of interest, there were no clusters that survived at a more liberal threshold of $p=0.005$ uncorrected. Outside the regions of interest, no activations survived multiple-comparison correction at the whole-brain level. Direct comparisons between effort and risk coding within each ROI (including all voxels contained within the anatomically defined regions) showed significantly stronger coding of effort rather than risk costs in SMA and putamen $(p=0.03, t=1.98$ and $p=$ $0.04, t=1.80$ respectively) and significantly stronger coding of risk rather than effort costs in the right, but not the left insula ( $p=0.03, t=1.90$ and $p=0.21, t=0.83$ respectively). These results support the notion that the brain codes effort and risk costs in a spatially separate manner when costs are presented in an isolated manner. Next we assessed whether this notion would also hold for compound trials, where participants are forced to integrate effort and risk.

\section{Activations relating to compound options}

We first asked whether the regions coding changes in effort and risk costs in isolated options would also code the changes in costs of the compound effort and risk options. We looked for activity in the insula and SMA correlating with the risk and effort levels separately during compound option presentation. Activity in the right insula correlated with risk during compound option presentation (peak at 33, 20,$13 ; p=0.04$ corrected for $15 \mathrm{~mm}$ sphere around the peak coordinate in isolated risk trials) and activity in the SMA tracked the effort during compound stimulus presentation (peak at $-9,17,40 ; p=$ 0.006 corrected for $15 \mathrm{~mm}$ sphere around the peak coordinate identified isolated effort trials).

Our behavioral analysis showed that costs during compound options could be best explained by a linear combination of effort and risk costs (Fig. $2 \mathrm{~F}$ ). We therefore investigated the anterior cingulate and frontal pole, regions that have previously been implicated in the integration of cognitive costs or of effort with other economic factors. This revealed distinct clusters of activity that correlated with the changes in the subjective costs of compound options in the frontal pole (Fig. $5 \mathrm{~A}$, peak at $-9,62,22 ; Z=3.58 ; p<0.05$ corrected for frontopolar region). Intriguingly, these regions did not correlate with subjective costs during isolated trials at the threshold used throughout (isolated effort trials, $p=0.62$; isolated risk trials, $p=0.84$ ), lending support to our alternative hypothesis that the brain codes costs in a distinct fashion when costs are presented separately but in a combined and integrated manner when costs are presented in compound.

Given the frontal pole coded costs preferentially in compound trials, the question arises whether and how these regions interact with the regions coding costs in isolated trials. Accordingly, we performed a psychophysiological interaction-based analysis (Park et al., 2011) to assess whether activity in the frontal pole during compound trials could be explained by activity in the regions coding risk (bilateral insula) and effort ( $\mathrm{pPut}$ and vSMA) 
during isolated trials. We hypothesized that the degree to which isolated risk and effort areas correlated with areas integrating costs during compound trials would be proportional to the participant-specific influence that risk attitude and effort attitude had on their combined valuations.

To test this hypothesis, we performed a multiple regression on the behavioral data to assess the relative impact of subjective risk and effort costs (as measured in isolated trials) on the subjective costs of the compound options (see Compound trials containing both effortful and risky options). We also performed an equivalent analysis using the time courses of the isolated effort and risk regions to assess their relative influence on the time course of the frontal pole. As hypothesized, the relative impact of effort versus risk on compound option valuation correlated with the relative degree to which individual effort (vSMA) versus risk (insula) regions were coupled with frontal pole activity $\left(R^{2}=0.55 ; p=0.01\right)$ (Fig. $\left.5 B\right)$.

\section{Discussion}

This study provides the first evidence that costs arising from physical effort and costs arising from risk are primarily processed in distinct brain regions. By placing physical effort costs and risk costs on the same scale (willingness to pay) we were able to make a direct comparison between the two at both behavioral and neural levels. Our behavioral results show that, although participants discounted high-effort and high-risk options in a similar manner, effort costs correlated primarily with activity in the pPut and vSMA, whereas risk costs correlated primarily with activity in the insula. Our experiment provided a novel twist to previous work on physical effort costs by including compound options that combined both risky and effortful aspects. For these options, costs correlated with activity in the frontal pole. These results suggest that the brain only codes costs in an integrated manner when it is necessary to do so, providing an explanation for the seemingly conflicting previous accounts of combined (Treadway et al., 2012) versus distinct (Prévost et al., 2010) cost coding in the brain.

In line with previous findings, our behavioral results indicate that participants discounted future reward value to greater extents as the effort (Prévost et al., 2010) required to attain the reward, or the reward-variability risk (Christopoulos et al., 2009), increased. However, a major goal of this study was to investigate how participants combined these two costs to arrive at subjective valuations for compound options that were both risky and effortful. Our results suggest that participants add the two different cost modalities in a linear manner, decomposing decision-relevant factors in a similar fashion to mean-variance models in economics (Markowitz, 1952). It should be noted, however, that in our experiment we did not vary the mean (objective) value of options, and participants did not make choices. We believe that these differences in task design caused our participants to focus primarily on costs as opposed to reward magnitude or cost-benefit trade-offs investigated in previous experiments (Croxson et al., 2009; Kurniawan et al., 2010). Although the BDM auction mechanism used to assess the subjective values of options is incentive-compatible and widely used in economics, the possibility remains that BDM valuations may not perfectly reflect preferences in choice situations (Horowitz, 2006).

We found spatially separate coding of risk in insula and effort in SMA and putamen. Although economic theory may predict

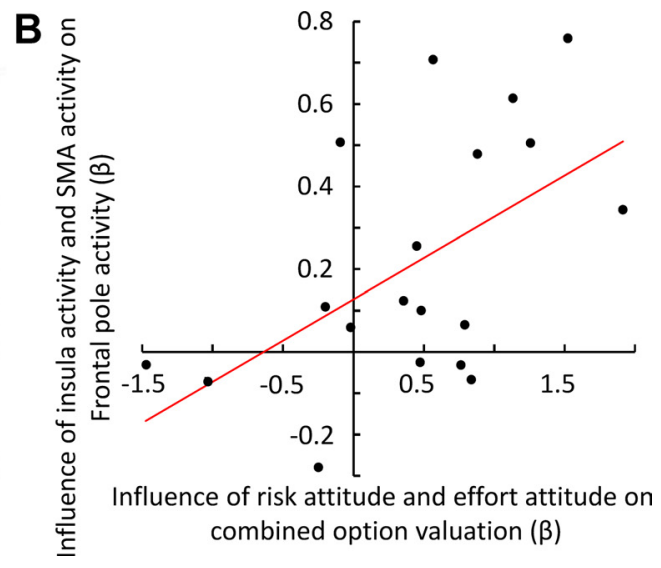

combined option valuation $(\beta)$

Figure 5. BOLD responses on compound effort and risk trials. $A$, Neural activity in the frontal pole (peak at $-9,62,22 ; Z=3.58$ $p<0.05$ corrected for frontopolar region) correlated with the subjective costs of compound stimuli at the time of option preseninsula (isolated risk region) on the frontal pole activity during compound trials could be explained by the relative behavioral $0.55 ; p=0.01)$.

that costs arising from different factors should be commonly represented to ensure efficient processing (Keeney and Raiffa, 1976; Wallenius et al., 2008), our results from isolated effortful and purely risky trials agree with previous work showing multiple subsystems coding different economic aspects of options in the environment (Christopoulos et al., 2009; Prévost et al., 2010; Burke and Tobler, 2011). Coding the costs arising from different modalities in distinct regions may be computationally efficient when it is necessary to track each type of cost separately. For example, physical effort costs require energetic responses from the decision maker that may directly impact physiological states. A foraging animal choosing to exploit a current patch or to move on to search for another patch may treat the physical effort costs of traveling differently from the risk costs associated with variable patch-reward density as the former may have a more direct impact on the current energy budget.

BOLD responses in the pPut and vSMA correlated strongly with the effort costs denoted in purely effortful stimuli. Previous studies implicated the striatum in coding anticipated future efforts (Pessiglione et al., 2007; Croxson et al., 2009). A role of posterior striatum in effort processing is borne out by the fact that corticostriatal connections from posterior medial motor areas are more likely to terminate there than in other regions of the striatum (Inase et al., 1996; Lehéricy et al., 2004). The separate coding of effort costs may also be related to effector-specific coding of action values (Gershman et al., 2009), as in our task effortful options denoted a potential requirement of a physical response by the participant whereas risky options did not require any response.

The distinct coding of risk costs in the insula demonstrated here agrees with several previous studies implicating the insula in the coding of different types of economic risk (Paulus et al., 2003; Preuschoff et al., 2008; Burke and Tobler, 2011). One potential explanation for the separate coding of risk costs (when presented in isolated trials) is that risk may need to be processed in a more flexible manner in the brain. While effort could be expected to discount future reward values in a consistent way (with costs imposed by the physical limitations of the body), an individual's response to future reward uncertainty may change quickly depending on the dynamics of the external or internal environment (Symmonds et al., 2010). Risk attitude has been shown to change within the time frame of a single experiment (Huber and Kunz, 2007) and there is also a variety of risk attitudes present in 
the subject samples undergoing neuroimaging experiments (Christopoulos et al., 2009; Tobler et al., 2009).

To reconcile the differences in the previous literature regarding combined versus distinct coding of different cost modalities, we also investigated the coding of costs in compound options that entailed both effortful and risky elements. We found that when participants were forced to integrate these two costs, activity in the frontal poles correlated with the combined costs. The frontal pole has been implicated in the integration of higher-level cognitive processes (Bunge et al., 2009) and the extensive dendritic arborization of the neurons in this region suggest they receive inputs from a wide variety of neurons throughout the prefrontal cortex and other association areas (Jacobs et al., 2001; Ramnani and Owen, 2004). Prabhakaran and colleagues (2000) show that the frontal pole integrates different types of information during a working memory task. More generally, it has been suggested that the necessity to integrate or concurrently process the outcomes of $\geq 2$ cognitive operations or alternative options is a primary driver of activity in this area (Ramnani and Owen, 2004; Boorman et al., 2009; Bunge et al., 2009). We extend these previous findings by showing that the frontal pole integrates information about costs when the need arises.

Interestingly, the frontal pole performed the integration by communicating during compound trials with regions involved in isolated cost coding in a subject-specific manner. In other words, the degree to which the vSMA (effort coding) and insula (risk coding) influenced frontal pole activity correlated significantly with participants' individual sensitivity to effort and risk costs. Together, our results indicate that the frontal pole may integrate different economic factors according to subjective relevance, but only when integration is necessary. This contributes to our understanding of the role of the frontal pole in integrating distinct types of information for goal-directed behavior.

\section{References}

Bohm P, Lindén J, Sonnegård P (1997) Eliciting reservation prices: Becker-DeGroot-Marshak mechanisms vs. markets. The Economic J 107:1079-1089. CrossRef

Boorman ED, Behrens TE, Woolrich MW, Rushworth MF (2009) How green is the grass on the other side? Frontopolar cortex and the evidence in favor of alternative courses of action. Neuron 62:733-743. CrossRef Medline

Bunge SA, Helskog EH, Wendelken C (2009) Left, but not right, rostrolateral prefrontal cortex meets a stringent test of the relational integration hypothesis. Neuroimage 46:338-342. CrossRef Medline

Burke CJ, Tobler PN (2011) Reward skewness coding in the insula independent of probability and loss. J Neurophysiol 106:2415-2422. CrossRef Medline

Christopoulos GI, Tobler PN, Bossaerts P, Dolan RJ, Schultz W (2009) Neural correlates of value, risk and risk aversion contributing to decision making under risk. J Neurosci 29:12574-12583. CrossRef Medline

Cléry-Melin ML, Schmidt L, Lafargue G, Baup N, Fossati P, Pessiglione M (2011) Why don't you try harder? An investigation of effort production in major depression. PLoS One 6:e23178. CrossRef Medline

Collier G, Levitsky DA (1968) Operant running as a function of deprivation and effort. J Comp Physiol Psychol 66:522-523. CrossRef Medline

Croxson PL, Walton ME, O'Reilly JX, Behrens TE, Rushworth MF (2009) Effort-based cost-benefit valuation and the human brain. J Neurosci 29: 4531-4541. CrossRef Medline

Floresco SB, Tse MT, Ghods-Sharifi S (2008) Dopaminergic and glutamatergic regulation of effort- and delay-based decision making. Neuropsychopharmacology 33:1966-1979. CrossRef Medline

Gershman SJ, Pesaran B, Daw ND (2009) Human reinforcement learning subdivides structured action spaces by learning effector-specific values. J Neurosci 29:13524-13531. CrossRef Medline

Horowitz JK (2006) The Becker-DeGroot-Marschak mechanism is not necessarily incentive compatible, even for non-random goods. Econ Lett 93:6-11

Huber O, Kunz U (2007) Time pressure in risky decision-making: effect on risk defusing. Psychol Sci 49:415-426.
Inase M, Sakai ST, Tanji J (1996) Overlapping corticostriatal projections from the supplementary motor area and the primary motor cortex in the macaque monkey: an anterograde double labeling study. J Comp Neurol 373:283-296. CrossRef Medline

Jacobs B, Schall M, Prather M, Kapler E, Driscoll L, Baca S, Jacobs J, Ford K, Wainwright M, Treml M (2001) Regional dendritic and spine variation in human cerebral cortex: a quantitative Golgi study. Cereb Cortex 11:558-571. CrossRef Medline

Keeney R, Raiffa H (1976) Decisions with multiple objectives: preferences and value tradeoffs. New York: Wiley.

Kennerley SW, Dahmubed AF, Lara AH, Wallis JD (2009) Neurons in the frontal lobe encode the value of multiple decision variables. J Cogn Neurosci 21:1162-1178. CrossRef Medline

Kurniawan IT, Seymour B, Talmi D, Yoshida W, Chater N, Dolan RJ (2010) Choosing to make an effort: the role of striatum in signaling physical effort of a chosen action. J Neurophysiol 104:313-321. CrossRef Medline

Lehéricy S, Ducros M, Krainik A, Francois C, Van de Moortele PF, Ugurbil K, Kim DS (2004) 3-D diffusion tensor axonal tracking shows distinct SMA and pre-SMA projections to the human striatum. Cereb Cortex 14:1302-1309. CrossRef Medline

Markowitz H (1952) Portfolio selection. J Finance 7:77-91. CrossRef

Mohr PN, Biele G, Heekeren HR (2010) Neural processing of risk. J Neurosci 30:6613-6619. CrossRef Medline

Park SQ, Kahnt T, Rieskamp J, Heekeren HR (2011) Neurobiology of value integration: when value impacts valuation. J Neurosci 31:9307-9314. CrossRef Medline

Paulus MP, Rogalsky C, Simmons A, Feinstein JS, Stein MB (2003) Increased activation in the right insula during risk-taking decision making is related to harm avoidance and neuroticism. Neuroimage 19:1439-1448. CrossRef Medline

Pessiglione M, Schmidt L, Draganski B, Kalisch R, Lau H, Dolan RJ, Frith CD (2007) How the brain translates money into force: a neuroimaging study of subliminal motivation. Science 316:904-906. CrossRef Medline

Prabhakaran V, Narayanan K, Zhao Z, Gabrieli JD (2000) Integration of diverse information in working memory within the frontal lobe. Nat Neurosci 3:85-90. CrossRef Medline

Preuschoff K, Quartz SR, Bossaerts P (2008) Human insula activation reflects risk prediction errors as well as risk. J Neurosci 28:2745-2752. CrossRef Medline

Prévost C, Pessiglione M, Météreau E, Cléry-Melin ML, Dreher JC (2010) Separate valuation subsystems for delay and effort decision costs. J Neurosci 30:14080-14090. CrossRef Medline

Ramnani N, Owen AM (2004) Anterior prefrontal cortex: insights into function from anatomy and neuroimaging. Nat Rev Neurosci 5:184-194. CrossRef Medline

Richards JB, Mitchell SH, De Wit H, Seiden L (1997) Determination of discount functions in rats with an adjusting-amount procedure. J Exp Anal Behav 67:353-366. CrossRef Medline

Rudebeck PH, Walton ME, Smyth AN, Bannerman DM, Rushworth MF (2006) Separate neural pathways process different decision costs. Nat Neurosci 9:1161-1168. CrossRef Medline

Schmidt L, Palminteri S, Lafargue G, Pessiglione M (2010) Splitting motivation: unilateral effects of subliminal incentives. Psychol Sci 21:977-983. CrossRef Medline

St Onge JR, Floresco SB (2010) Prefrontal cortical contribution to riskbased decision making. Cereb Cortex 20:1816-1828. CrossRef Medline

Symmonds M, Emmanuel JJ, Drew ME, Batterham RL, Dolan RJ (2010) Metabolic state alters economic decision making under risk in humans. PLoS One 5:e11090. CrossRef Medline

Tobler PN, Christopoulos GI, O’Doherty JP, Dolan RJ, Schultz W (2009) Risk-dependent reward value signal in human prefrontal cortex. Proc Natl Acad Sci U S A 106:7185-7190. CrossRef Medline

Treadway MT, Buckholtz JW, Cowan RL, Woodward ND, Li R, Ansari MS, Baldwin RM, Schwartzman AN, Kessler RM, Zald DH (2012) Dopaminergic mechanisms of individual differences in human effort-based decision-making. J Neurosci 32:6170-6176. CrossRef Medline

Wallenius J, Dyer JS, Fishburn PC, Steuer RE, Zionts S, Deb K (2008) Multiple criteria decision making, multiattribute utility theory: recent accomplishments and what lies ahead. Manage Sci 54:1336-1349. CrossRef

Walton ME, Kennerley SW, Bannerman DM, Phillips PE, Rushworth MF (2006) Weighing up the benefits of work: behavioral and neural analyses 
of effort-related decision making. Neural Netw 19:1302-1314. CrossRef Medline

Walton ME, Groves J, Jennings KA, Croxson PL, Sharp T, Rushworth MF, Bannerman DM (2009) Comparing the role of the anterior cingulate cortex and 6-hydroxydopamine nucleus accumbens lesions on operant effort-based decision making. Eur J Neurosci 29:1678-1691. CrossRef Medline

Wunderlich K, Beierholm UR, Bossaerts P, O’Doherty JP (2011) The human prefrontal cortex mediates integration of potential causes behind observed outcomes. J Neurophysiol 106:1558-1569. CrossRef Medline 